\title{
A comparison of two methods for quantifying parasitic nematode fecundity
}

\author{
Lauren V. Austin ${ }^{1,2} \cdot$ Sarah A. Budischak ${ }^{1,3}$ • Jessica Ramadhin ${ }^{4} \cdot$ Eric P. Hoberg ${ }^{5}$. \\ Art Abrams $^{5}$ • Anna E. Jolles ${ }^{6}$ - Vanessa O. Ezenwa ${ }^{1,4}$
}

Received: 26 January 2017 / Accepted: 21 March 2017 / Published online: 29 March 2017

(C) Springer-Verlag Berlin Heidelberg 2017

\begin{abstract}
Accurate measures of nematode fecundity can provide important information for investigating parasite life history evolution, transmission potential, and effects on host health. Understanding differences among fecundity assessment protocols and standardizing methods, where possible, will enable comparisons across different studies and host and parasite species and systems. Using the trichostrongyle nematode Cooperia fuelleborni isolated from wild African buffalo (Syncerus caffer), we compared egg recovery and enumeration between two methods for measuring the fecundity of female worms. The first method, in utero egg count, involves visual enumeration of the eggs via microscopic inspection of the uterine system. The second method, ex utero egg count, involves dissolving the same specimens from above in a sodium chloride solution to release the eggs from the female's uterus, then enumeration under an inverted microscope. On
\end{abstract}

Sarah A. Budischak

sbudischak@princeton.edu

1 Odum School of Ecology, University of Georgia, 140 E. Green St, Athens, GA 30602, USA

2 Department of Fish and Wildlife Conservation, Virginia Polytechnic Institute and State University, 310 West Campus Drive, Blacksburg, VA 24061, USA

3 Department of Ecology and Evolutionary Biology, Princeton University, 106A Guyot Hall, Princeton, NJ 08544-2016, USA

4 Department of Infectious Diseases, University of Georgia, 510 D.W. Brooks Drive, Athens, GA 30602, USA

5 United States Department of Agriculture, Agricultural Research Service, Beltsville, MD, USA

6 Department of Biomedical Sciences and Department of Integrative Biology, Oregon State University, 105 Magruder Hall, Corvallis, OR 97331, USA average, the ex utero method resulted in $34 \%$ more eggs than the in utero method. However, results indicate that the two methods used to quantify parasitic nematode fecundity are highly correlated. Thus, while both methods are viable options for estimating relative nematode fecundity, we recommend caution in undertaking comparative studies that utilize egg count data collected using different methods.

Keywords Fecal egg count $\cdot$ Helminth fecundity $\cdot$ Cooperia fuelleborni $\cdot$ Trichostrongyle nematode

\section{Introduction}

Parasitic nematodes infect the gastrointestinal (GI) tracts of nearly all vertebrates (Poulin 1995; Grencis et al. 2014), and prevalence can be high in wild populations (Shaw and Dobson 1995; Koprivnikar et al. 2012). In addition to causing direct mortality (Stjernman et al. 2008), GI nematodes can also have a number of sub-lethal effects on hosts that impact fitness. For example, infection with Ostertagia gruehneri Skrjabin, 1929, led to reductions in host body mass, back fat depth, and the probability of pregnancy in Svalbard reindeer Rangifer tarandus platyghynchus Vrolik, 1829, with ultimate effects on host fitness (Stien et al. 2002; Albon et al. 2002). Often, the fitness costs of GI nematode infection are a direct result of the number of worms harbored by a host (Hudson et al. 1992; Stjernman et al. 2008; Benesh 2011). However, fitness costs to the host have also been linked to the number of eggs produced by nematodes, i.e., their total fecundity (Rowe et al. 2008; Holand et al. 2015). For example, Holand et al. (2015) found that the number of gapeworm Syngamus trachea (Montagu, 1811) eggs in the parent bird's feces was negatively correlated with the proportion of successful fledglings in house sparrows (Passer domesticus, Linnaeus, 1758). Quantifying nematode fecundity, in addition to adult 
worm burdens, can therefore provide valuable insight into the costs of infection, patterns of dissemination in the environment, and the dynamics of local transmission.

From the parasite perspective, fecundity is central to determining how many offspring each individual will contribute to the next generation (Godfray et al. 1991). Correspondingly, parasite fecundity may be a trait under strong selection (Skorping et al. 1991; Poulin 1995, 1997). Indeed, the lifecycle of GI nematodes typically require that infective stages (eggs or larvae) leave the host in order to generate new infections. Thus, transmission of GI nematodes depends on the production of viable eggs, their development in the environment, dispersal of infective stages and potential for exposure of appropriate hosts. Production of eggs can depend on factors such as parasite body size, the availability of host resources (i.e., space and nutrients), and host immunity, that affect how much energy female nematodes can devote to egg production (Poulin 1997; Singleton et al. 2011; Romeo et al. 2014). Quantifying nematode fecundity can provide valuable insight into transmission potential, the strength of helminth competition, parasite evolutionary ecology, and the life history strategies of parasites.

There are several methods for measuring the fecundity of female nematodes, each having advantages and disadvantages in terms of cost, training, and sample preservation. In cases of non-invasive sampling of the host, total fecundity of the community of worms infecting a host can be quantified by counting worm eggs in host fecal samples (e.g., fecal flotation or sedimentation (Faust et al. 1938; Coles et al. 1992). Fecal egg counting methods, however, do not provide information on faunal diversity (species cannot usually be identified) and adult worm burdens, so per capita estimates of fecundity cannot be calculated, which limits the value of this approach for studies focused on understanding worm fecundity from a parasite perspective. When hosts are sampled through field-based collection and adult worms are isolated from the GI tract, there are two common protocols for measuring individual worm fecundity. The first method involves clearing the worm specimen (a process that renders the external cuticle transparent) and visual enumeration of the eggs via inspection of the uterine system (single, paired, or multiple tracts, depending on the taxon involved) under magnification (hereafter, in utero count) (Karvonen et al. 2006). Notably, this in utero method is non-destructive, which allows specimens to be retained for archival storage, but requires a skilled professional; further, applicability may be limited by the specific structure of the uterus and whether it is narrow and tubular with relatively few discrete eggs or broad and voluminous with numerous and overlapping eggs. A modified version of this technique involves removing the reproductive tract and extracting the eggs before counting under light microscopy (Kuzmina et al. 2012). This modified technique enhances the observer's ability to accurately count eggs, but results in the destruction of the specimen. The second method of assessing fecundity involves the chemical digestion of the female nematode's tissues to isolate the eggs (hereafter, ex utero count) (Kanobana et al. 2002). This ex utero method allows for easy visualization and enumeration of eggs, but results in the destruction of both the specimen and the eggs. In this study, we compared egg recovery and enumeration between the in utero (Karvonen et al. 2006) and ex utero (Kanobana et al. 2002) methods of fecundity assessment. We focused on Cooperia fuelleborni Hung, 1926, isolated from wild African buffalo Syncerus caffer Sparrman, 1779.

\section{Methods}

\section{Sample collection}

Adult nematode specimens were collected from African buffalo in Kruger National Park, South Africa, between June and August 2012. At the end of a 4-year study on parasite interactions (Ezenwa and Jolles 2015), buffalo were euthanized following the South African National Parks Standard Operating Procedure for Lethal Population Control. Aliquots representing $2.5 \%$ of the contents of the small intestine were collected following standard procedures (Wood et al. 1995) and preserved in 5\% phosphate buffered formalin as described in Budischak et al. (2015). Adult nematode specimens were isolated by rinsing the samples through 250 and $44 \mu \mathrm{m}$ sieves. Specimens were stored in $70 \%$ ethanol and identified at the USDA Agricultural Research Service, US National Parasite Collection (USNPC), and Animal Parasitic Disease Laboratory. We chose to focus on C. fuelleborni since it was the most prevalent (94\% of hosts) and abundant (96\% of specimens) GI nematode in the study population (Budischak et al. 2015). In utero and ex utero methods were applied to between 4 and 10 (mean \pm se $8.2 \pm 0.7$ ) female C. fuelleborni specimens collected from each of ten buffalo. In total, 82 individual parasite specimens were examined.

\section{In utero fecundity}

To quantify female fecundity in utero, we visually inspected and counted the number of eggs within the uterine tract of each C. fuelleborni specimen. To enhance visualization, specimens were first cleared using a phenol-alcohol mixture consisting of 80 parts melted phenol crystals and 20 parts absolute ethanol. In utero counts were done at $400 \times$ in differential interference contrast on a Zeiss Axiophot scope. Counts were based on observations from single intact specimens starting with the first fully shelled, free, eggs in the anterior and posterior uterine limbs of each specimen, from the respective ovarian limb and including the anterior and posterior ovejector and vestibule, consistent with structure of the female system in these trichostrongyline nematodes. 
Table 1 With the outliers included, ex utero egg count was still a significant predictor of the percent difference between ex utero and in utero fecundity methods. The random effect of buffalo identity explains a larger (15\%), but still minimal, fraction of variance in percent difference

\begin{tabular}{llllll}
\hline & Estimate & $\mathrm{SE}$ & $\mathrm{df}$ & $t$ value & $p$ value \\
Ex utero count & 0.73 & 0.14 & 79.5 & 5.08 & $<0.0001$ \\
& Variance & $\mathrm{SD}$ & & & \\
Buffalo ID & 75.3 & 8.7 & & & \\
Residual & 423.0 & 20.6 & & & \\
\hline
\end{tabular}

\section{Ex utero fecundity}

To quantify female fecundity using the ex utero method, we dissolved the same specimens from above in bleach following the methods of Kanobana et al. (2002). Specimens were processed in $50 \mu \mathrm{L}$ of $5 \%$ sodium hyperchlorite (Sigma Aldrich, USA) to release the eggs from the female's uterus. After approximately $4 \mathrm{~min}$ in solution, the specimen tissue dissolved and the eggs were released. Because of their protective shells, eggs remained stable for an additional $\sim 2 \mathrm{~min}$, allowing an observer to perform egg counts before dissolution of the eggs. A 5\% sodium hypochlorite concentration was selected after preliminary trials, which is a modification of the methods used by Kanobana et al. (2002). We used an inverted stage microscope (Olympus CKX41) at $100 \times$ power, to visualize the eggs. Each set of eggs was counted three times by a single observer. The best estimate of the replicate counts for a specimen was used as the ex utero egg count.

\section{Statistical analysis}

First, the correlation between egg counts derived from the in utero and ex utero measurement techniques was examined using a Pearson's correlation test. To explore whether host identity influenced the degree of variability between the two methods, buffalo identity was tested as a predictor of the difference in the ex utero versus in utero egg counts using an ANOVA. Next, a general linear mixed model (GLMM) that accounted for the sampling of multiple female worms per host individual was used to test for an effect of number of eggs on the magnitude of the difference between counts (while controlling for host identity). The percent difference between ex utero and in utero egg count for each specimen was the response variable in the GLMM, ex utero egg count was included as a fixed effect, and host identity was included as a random effect.

Three outliers were excluded from all of the analyses. The outliers occurred in worms counted on the first/early days of data collection and were likely the result of a newly trained technician. Thus, a sample was considered appropriate to remove if it was not only a statistical outlier but was also counted on an early observation day. Removing these outliers allowed model residuals to meet normality assumptions but did not qualitatively change results (Table 1).

\section{Results}

The ex utero and in utero methods for assessing fecundity were strongly and positively correlated $(n=79, r=0.80$, $p<0.0001$, Fig. 1). However, for a vast majority of specimens (95\%), the ex utero count was higher than the in utero count. On average, the ex utero method resulted in $34.2 \%$ $(18.5 \pm 1.14)$ more eggs than the in utero method. Minimum, maximum, and mean egg counts are presented in Table 2. Differences in egg count between methods did not vary by host (ANOVA $F=1.09, \mathrm{df}=9,69, p=0.38$; Fig. 2 ). However, the percent difference between counting methods was affected by ex utero nematode fecundity (GLMM: Est $\pm \mathrm{SE}=0.45 \pm 0.12, t=3.61, \mathrm{df}=51, p=0.00069$;

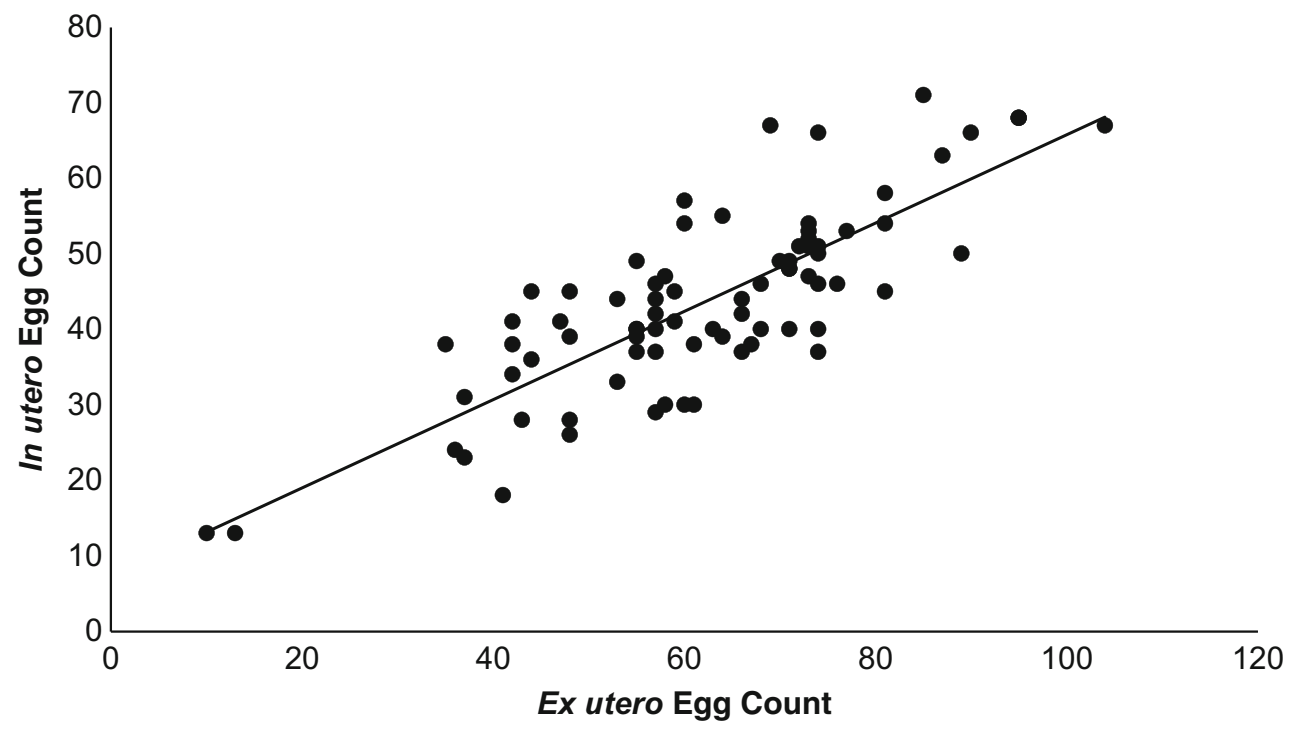

Fig. 1 Estimates of fecundity for each C. fuelleborni female were correlated between the two counting methods 
Table 2 Minimum, maximum, and mean egg counts and standard deviation for the ex utero and in utero egg counting methods, with outliers excluded

\begin{tabular}{lcl}
\hline & Ex utero & In utero \\
\hline Minimum & 10 & 13 \\
Maximum & 104 & 71 \\
Mean & 62.04 & 43.57 \\
Standard deviation & 16.81 & 12.19 \\
\hline
\end{tabular}

Fig. 3). The random effect of host identity explained less than $3 \%$ of the variance in the model and was not significant (GLMM: buffalo ID: variance $\pm \mathrm{SE}=9.4 \pm 3.1$; residual: variance $\pm \mathrm{SE}=319 \pm 18$ ).

\section{Discussion}

Results indicate that the two methods used to quantify parasitic nematode fecundity are highly correlated, suggesting that both methods are viable options for estimating relative nematode fecundity within a study. However, the ex utero method consistently recovered a higher number of eggs. The ex utero method resulted in approximately 18.5 (34.2\% difference) more eggs on average than the in utero method, which suggests that in utero counts may underestimate nematode fecundity. Moreover, percent difference was positively correlated with ex utero egg count, indicating a greater discrepancy between techniques for individuals with high fecundity. Due to these differences between techniques, comparisons across studies using these different fecundity assessment methodologies are likely to be biased.
The lower egg counts observed in the in utero counts may be due, in part, to the protocol we used for counting eggs in utero. In utero counting began by looking upstream of the ovejectors for the point between adherent eggs (i.e., shelled but not fully developed) and independent eggs. Since it is difficult to distinguish adherent eggs from developing ova visually, only independent eggs from that point to the vulva were counted. By contrast, the ex utero counts included both adherent and independent eggs because, unlike developing ova, both have shells resistant to the bleach solution. The inclusion of adherent eggs in ex utero, but not in utero counts, could account for some of the difference between the two methods.

The appropriate nematode fecundity assessment method to use in any given situation depends on the advantages and disadvantages of each method as well as study logistics, including personnel training, sample preservation or archiving needs, and time. For example, the in utero method requires highly trained personnel to count eggs while they are still in utero, but allows for the archival preservation of specimens. This method might be better suited for use in studies that prioritize specimen preservation and involve species with a relatively restricted and strongly tubular uterus, and in which the eggs are organized in a linear fashion, rather than in layers, or with a large number of overlapping eggs. For example, good candidate taxa among ungulate nematodes for the in utero method include Trichostrongylina such as species among the Cooperiinae, Trichostrongylinae, Ostertagiinae, and some Nematodirinae (e.g., Skrjabin et al. 1954; Hoberg et al. 2005, 2012). Conversely, the ex utero method could provide more reliable counts for species whose eggs are difficult to count in utero, including taxa among the Trichostrongyloidea such as Ostertagiinae with species of Africanastrongylus Hoberg et al., 2008, and Robustostrongylus Hoberg et al., 2009, and

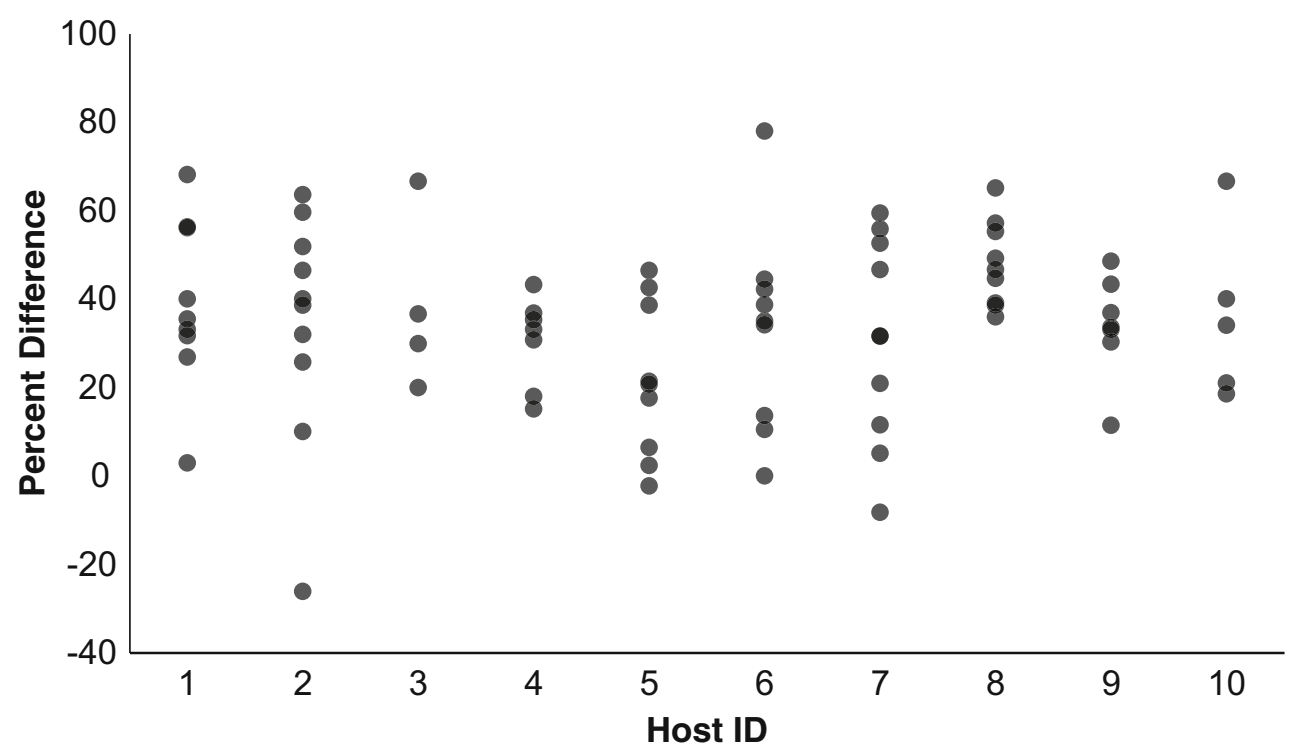

Fig. 2 Percent difference between counting methods did not vary among hosts 
Fig. 3 Percent difference between fecundity methods was positively associated with ex utero fecundity

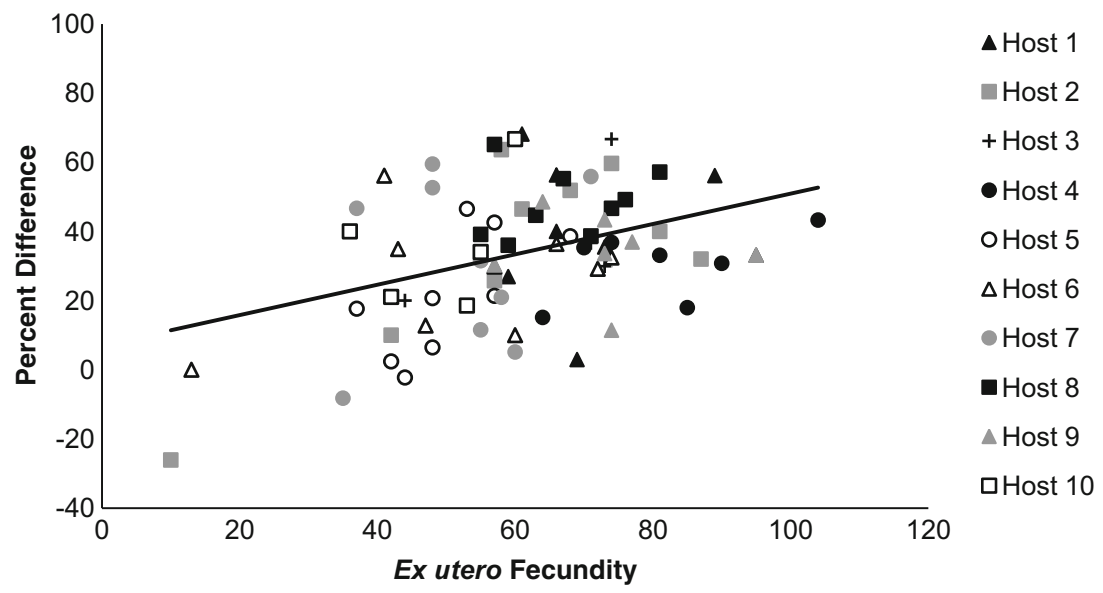

Haemonchinae with species of Haemonchus Cobb, 1898, and Ashworthius Le Roux, 1930, (Chauhan et al. 1972; Lichtenfels et al. 2002).

In contrast to the in utero method, the ex utero method requires minimal personnel training, but specimens are destroyed in the process. As such, this method might only be appropriate for studies where tissue and specimens do not have to be archived or used for other purposes or where species identity is considered to be unequivocal; in these situations, it remains a critical point to retain voucher specimens held in a museum repository. In addition, accuracy in the ex utero methods depends on performing counts within a short window of time. Counts must occur not too soon or too late after exposure to sodium hypochlorite solution (i.e., during an approximately 2 -min window), which could pose a challenge for novice technicians, as demonstrated by some initial counts in our analyses that resulted in outliers.

Accurate measures of nematode fecundity can provide important information for investigating parasite life history evolution (Skorping et al. 1991), transmission potential (May and Anderson 1979), and effects on host health (Stien et al. 2002; Albon et al. 2002; Holand et al. 2015). This study compared two methods for assessing fecundity of a trichostrongyle nematode, finding that both were good options, although each had its unique advantages and disadvantages. However, because of the large discrepancy in egg counts between methods, we recommend caution in undertaking comparative studies and meta-analyses that utilize egg count data from multiple studies. Understanding the correspondence between different protocols for assessment of nematode fecundity, and standardizing methods, where possible, will enable comparisons across different studies and host and parasite species and systems.

Acknowledgements Many thanks to South Africa National Parks Veterinary Services for assistance with the animal capture operations. We thank R. Spaan, J. Spaan, A. Majewska, J. Alagappan, C. Becker, B. Beechler, E. Belinfante, E. Gorsich, C. Gondhalekar, C. Hebbale, T.
Lavelle, L. Leathers, L. Megow, T. Mowla, A. Petrelli, K. Raum, N. Rogers, K. Sakamoto, P. Snyder, and M. Smith for invaluable assistance in the field and in the lab. All animal protocols were approved by the UGA Institutional Animal Care and Use Committee (AUP\#: A2013 08017-Y1-A0; AUP\#: A2010 10-190-Y3-A5). This work was funded by the National Science Foundation Ecology of Infectious Diseases Grant to VOE and AEJ (DEB-1102493, EF-0723928). LVA was supported by a Georgia Museum of Natural History's Joshua Laerm Academic Support Award for Undergraduate Research and an NSF REU supplement to DEB-1102493; JR was supported by the NSF Population of Infectious Diseases REU Program at the University of Georgia (DBI-1156707).

\section{References}

Albon SD, Stien A, Irvine RJ, Langvatn R, Ropstad E, Halvorsen O (2002) The role of parasites in the dynamics of a reindeer population. Proc R Soc Lond Ser B-Biol Sci 269:1625-1632. doi:10.1098/ rspb.2002.2064

Benesh DP (2011) Intensity-dependent host mortality: what can it tell us about larval growth strategies in complex life cycle helminths? Parasitology 138:913-925. doi:10.1017/S0031182011000370

Budischak SA, Hoberg EP, Abrams A, Jolles AE, Ezenwa VO (2015) A combined parasitological molecular approach for noninvasive characterization of parasitic nematode communities in wild hosts. Mol Ecol Resour 15:1112-1119. doi:10.1111/1755-0998.12382

Chauhan PPS, Pande BP, Singh M (1972) A new species of Ashworthius Le Roux, 1930 (Haemonchinae: Trichostrongylidae) from two wild ruminants with a note on associated lesions. J Helminthol 46:149 155. doi:10.1017/S0022149X00022239

Coles GC, Bauer C, Borgsteede FHM, Geerts S, Klei TR, Taylor MA (1992) World Association for the Advancement of Veterinary Parasitology (W.A.A.V.P) methods for the detection of anthelmintic resistance in nematodes of veterinary importance. Vet Parasitol 44: 35-44. doi:10.1016/0304-4017(92)90141-U

Ezenwa VO, Jolles AE (2015) Opposite effects of anthelmintic treatment on microbial infection at individual versus population scales. Science 347:175-177. doi:10.1126/science.1261714

Faust EC, D'Antoni JS, Odom V, Miller MJ, Peres C, Sawitz W, Thomen LF, Tobie J, Walker JH (1938) A critical study of clinical laboratory technics for the diagnosis of protozoan cysts and helminth eggs in feces. AmJTrop Med Hyg 18:169-183

Godfray HCJ, Partridge L, Harvey PH (1991) Clutch size. Annu Rev Ecol Syst 22:409-429. doi:10.1146/annurev.es.22.110191.002205 
Grencis RK, Humphreys NE, Bancroft AJ (2014) Immunity to gastrointestinal nematodes: mechanisms and myths. Immunol Rev 260:183205. doi:10.1111/imr.12188

Hoberg EP, Lichtenfels JR, Rickard LG (2005) Phylogeny for genera of Nematodirinae (Nematoda: Trichostrongylina). J Parasitol 91:382 389. doi:10.1645/GE-3408

Hoberg E, Abrams A, Pilitt PA, Jenkins EJ (2012) Discovery and description of a new trichostrongyloid species (Nematoda: Ostertagiinae), abomasal parasites in mountain goat, Oreamnos americanus, from the western cordillera of North America. J Parasitol 98:817-846

Holand H, Jensen H, Tufto J, Parn H, Saether BE, Ringsby TH, Cimmaruta R (2015) Endoparasite infection has both short- and long-term negative effects on reproductive success of female house sparrows, as revealed by faecal parasitic egg counts. PLoS One 10: e0125773. doi:10.1371/journal.pone. 0125773

Hudson PJ, Newborn D, Dobson AP (1992) Regulation and stability of a free-living host-parasite system: Trichostrongylus tenuis in red grouse. I. Monitoring and parasite reduction experiments. J Anim Ecol 61:477-486. doi:10.2307/5338

Kanobana K, Ploeger HW, Vervelde L (2002) Immune expulsion of the trichostrongylid Cooperia oncophora is associated with increased eosinophilia and mucosal IgA. Int J Parasitol 32:1389-1398. doi:10. 1016/S0020-7519(02)00132-7

Karvonen A, Cheng G-H, Seppälä O, Valtonen ET (2006) Intestinal distribution and fecundity of two species of Diplostomum parasites in definitive hosts. Parasitology 132:357-362. doi:10.1017/ S0031182005009091

Koprivnikar J, Marcogliese DJ, Rohr JR, Orlofske SA, Raffel TR, Johnson PTJ (2012) Macroparasite infections of amphibians: what can they tell us? EcoHealth 9:342-360. doi:10.1007/s10393-0120785-3

Kuzmina TA, Lyons ET, Tolliver SC, Dzeverin II, Kharchenko VA (2012) Fecundity of various species of strongylids (Nematoda: Strongylidae)-parasites of domestic horses. Parasitol Res 111: 2265-2271. doi:10.1007/s00436-012-3077-5

Lichtenfels JR, Pilitt PA, Gibbons LM, Hoberg EP (2002) Redescriptions of Haemonchus mitchelli and Haemonchus okapiae (Nematoda: Trichostrongyloidea) and description of a unique synlophe for the Haemonchinae. J Parasitol 88:947-960. doi:10.1645/00223395(2002)088[0947:ROHMAH]2.0.CO;2

May RM, Anderson RM (1979) Population biology of infectious diseases: part II. Nature 280:455-461. doi:10.1038/280455a0
Poulin R (1995) Clutch size and egg size in free-living and parasitic copepods: a comparative analysis. Evolution 49:325-336. doi:10. 2307/2410343

Poulin R (1997) Egg production in adult trematodes: adaptation or constraint? Parasitology 114:195-204. doi:10.1017/S0031182096008372

Romeo C, Wauters LA, Cauchie S, Martinoli A, Matthysen E, Saino N, Ferrari N (2014) Faecal egg counts from field experiment reveal density dependence in helminth fecundity: Strongyloides robustus infecting grey squirrels (Sciurus carolinensis). Parasitol Res 113: 3403-3408. doi:10.1007/s00436-014-4005-7

Rowe A, McMaster K, Emery D, Sangster N (2008) Haemonchus contortus infection in sheep: parasite fecundity correlates with worm size and host lymphocyte counts. Vet Parasitol 153:285-293. doi:10. 1016/j.vetpar.2008.01.040

Shaw DJ, Dobson AP (1995) Patterns of macroparasite abundance and aggregation in wildlife populations: a quantitative review. Parasitology 111:S111-S133. doi:10.1017/S0031182000075855

Singleton DR, Stear MJ, Matthews L (2011) A mechanistic model of developing immunity to Teladorsagia circumcincta infection in lambs. Parasitology 138:322-332. doi:10.1017/S0031182010001289

Skorping A, Read AF, Keymer AE (1991) Life history covariation in intestinal nematodes of mammals. Oikos 60:365. doi:10.2307/ 3545079

Skrjabin KI, Shikobalova NP, Shul'tz RS (1954) Trichostrongylids of animals and man. Osnovy Nematodologii III. Isdatel'stvo Akademii Nauk SSSR. English Translation, 1960, US Department of Agriculture and Israel Program for Scientfic Translations, Jerusalem, Moskva

Stien A, Irvine RJ, Ropstad E, Halvorsen O, Langvatn R, Albon SD (2002) The impact of gastrointestinal nematodes on wild reindeer: experimental and cross-sectional studies. J Anim Ecol 71:937-945. doi:10.1046/j.1365-2656.2002.00659.x

Stjernman M, Råberg L, Nilsson J-Å (2008) Maximum host survival at intermediate parasite infection intensities. PLoS One 3:e2463. doi: 10.1371/journal.pone.0002463

Wood IB, Amaral NK, Bairden K, Duncan JL, Kassai T, Malone JB, Pankavich JA, Reinecke RK, Slocombe O, Taylor SM, Vercruysse J (1995) World Association for the Advancement of Veterinary Parasitology (WAAVP) of guidelines for evaluating the efficacy of anthelmintics in ruminants (bovine, ovine, caprine). Vet Parasitol 58:181-213. doi:10.1016/0304-4017(95)00806-2 